\title{
Entre el orden, el vínculo, el conflicto y el poder. Aproximación histórica al concepto de control social y análisis de sus usos historiográficos
}

Resumen: Este artículo pretende contribuir a una mayor claridad alrededor del concepto de control social; el cual es usado frecuentemente, pero cuya historicidad no siempre es considerada. Con este fin, se revisaron obras que lo desarrollan teóricamente de diferentes formas: como función, como generador del vínculo social, asociado a la resolución de conflictos y como forma de poder. Posteriormente se analizan algunos trabajos históricos que usan el concepto. El artículo estudia en primer lugar el desenvolvimiento histórico del concepto, para luego pasar al examen de algunas de las formas en que ha sido aplicado para analizar los órdenes sociales pasados.

Palabras clave: control social, vínculo social, conflicto, orden social, legitimidad.

\section{Between order, social bond, conflict, and power. A historical approach to the concept of social control and an analysis of its historiographic uses}

Abstract: This article contributes to greater clarity around the concept of social control, a concept used frequently, but whose own historicity is not always taken into account. To this end, in the first place, I review literature that has developed the concept theoretically: as a function, as a generator of social bonds, associated to conflict resolution, and as a form of power. Subsequently, I analyze historical works that use the concept, addressing its historical development and the ways in which it has been employed to study past social orders.

Keywords: social control, social bond, conflict, social order, legitimacy.

\section{Entre ordem, vínculo social, conflito e poder. Abordagem histórica do conceito de controle social e análise dos seus usos historiográficos}

Resumo: Este artigo pretende contribuir para uma maior clareza conceitual em torno do conceito de controle social, conceito de uso frequente, mas cuja própria historicidade nem sempre é considerada. Para este fim, revisamos trabalhos que o desenvolvem teoricamente de diferentes formas: como função, como gerador de vínculo social, associado à resolução de conflitos e como forma de poder. Posteriormente, alguns trabalhos históricos que usam o conceito são analisados. 0 artigo primeiro estuda o desenvolvimento histórico do conceito e, em seguida, examina algumas das maneiras pelas quais ele foi aplicado para analisar as ordens sociais passadas.

Palavras-chave: controle social, vínculo social, conflito, ordem social, legitimidade.

Cómo citar este artículo: Carlos Alberto Toro Silva, “Entre el orden, el vínculo, el conflicto y el poder. Aproximación histórica al concepto de control social y análisis de sus usos historiográficos", Trashumante. Revista Americana de Historia Social 14 [2019]: 174-194.

DOI: 10.17533/udea.trahs.n14a08

Fecha de recepción: 3 de abril de 2018

Fecha de aprobación: 4 de diciembre de 2018

Carlos Alberto Toro Silva: Doctorando en Historia de la Universidad de los Andes.

Correo electrónico: ca.toro964@uniandes.edu.co 


\section{Entre el orden, el vínculo, el conflicto y el poder. Aproximación histórica al concepto de control social y análisis de sus usos historiográficos}

Carlos Alberto Toro Silva

Introducción

El concepto de control social sirve para aproximarse al problema de la constitución Ey conservación del orden social. Este concepto, surgido en la sociología estadounidense, ha influido en la historiografía social. Así lo muestra Pedro Oliver Olmo en su análisis de algunas de las maneras en que este cobra sentidos concretos en investigaciones encaminadas a "aprehender la dinámica del orden como las formas de control del desorden y la conflictividad". ${ }^{1}$ El presente artículo sigue una línea similar, aunque enfocado en el desarrollo que se le ha dado en Estados Unidos y algunas de sus aplicaciones en la historiografía estadounidense y latinoamericana.

Una idea central en este artículo es que en la sociología clásica y la criminología estadounidenses el control social tiende a referirse a la función de mantener o constituir un orden social, cuya legitimidad es tomada como presupuesto o necesidad. Por el contrario, en las teorías revisionistas como la de Michel Foucault (1926-1984) es ante todo de una forma de poder. La teoría de Donald Black (1941) lo asimila a la resolución de conflictos. Algunas investigaciones históricas utilizan las formas clásicas del concepto; otras le dan distintas connotaciones, y llegan a cuestionar la presunción de un único orden social legítimo. En otras investigaciones el control social aparece vinculado a una perspectiva conflictiva sobre la sociedad, aunque pueden reconocer la posibilidad de su coexistencia con otros órdenes sociales.

El artículo comenzará con una revisión de las teorías clásicas de la sociología estadounidense, primeras en hacer uso del concepto como fundamento del orden social, y de las teorías revisionistas posteriores. En segundo lugar, se explorarán los planteamientos de Foucault sobre el control, por cuanto hay historiadores que han tomado su obra como punto de referencia para dar forma a la problemática del

1. Pedro Oliver Olmo, "El concepto de control social en la historia social: estructuración del orden y respuestas al desorden", Historia Social 51 (2005): 91. 
control social. Más adelante, en la sección de análisis de los textos historiográficos, se prestará especial atención a la relación entre el control social y el orden social, para terminar con unas breves conclusiones. Debe anotarse que con el anterior ejercicio no se pretende hacer un recuento de todo lo escrito sobre el tema en el campo de la historiografia, sino presentar un análisis crítico sobre un concepto de uso común en las ciencias sociales. Con este fin, se examinó un conjunto de textos históricos estadounidenses y latinoamericanos, escogidos por su claridad a la hora de mostrar algunas de las formas en que las diferentes conceptualizaciones sobre el control social han sido utilizadas para estudiar órdenes sociales pasados.

\section{Desarrollos teóricos del control social}

¿Qué es el control social? Aunque sus definiciones son diversas, puede proponerse una inicial: es la garantía del cumplimiento de las normas que rigen las acciones de los miembros de un grupo social. La existencia de normas en un grupo humano implica regularidad en sus relaciones sociales. Aquí aparece la idea de orden social en un sentido sociológico, según la definición de Max Weber (1864-1920). ${ }^{2}$ Cuando los miembros del grupo aceptan como obligatorias las normas que rigen sus acciones se habla de un orden legítimo. En este orden de ideas, la legitimidad es el reconocimiento de la obligatoriedad de las normas de un orden social. Aunque el término legitimidad no necesariamente aparezca en las obras referidas al control social, la idea de la aceptación de las normas como presupuesto sí aparece en gran parte de ellas. En otras puede entenderse como un resultado del control.

El problema del orden social interesó a la sociología clásica como muestran las obras del mencionado Max Weber y Émile Durkheim (1858-1917); sin embargo, el concepto de control social surgió en el contexto particular de Estados Unidos a finales del siglo XIX. ${ }^{3}$ Las primeras conceptualizaciones sociológicas sobre el control social presuponían la legitimidad de ese orden social y en ellas el término tenía una connotación prescriptiva. En esto coinciden desde la primera y más amplia teoría de Edward Alsworth Ross (1866-1951) hasta las versiones más extremas de la teoría criminológica del control en las que el vínculo entre el orden social y el control social no es tan explícito. Antes de desarrollar estos planteamientos, es necesario responder a la pregunta de por qué el concepto de control social surgió precisamente en Estados Unidos.

Peter Spierenburg (1948), Morris Janowtiz (1919-1988) y Dario Melossi (1948) han dado elementos para responder a dicha pregunta. ${ }^{4}$ Según Spierenburg, Edward Alsworth Ross consideraba que el control social serviría “para promover el

2. Max Weber, Conceptos sociológicos fundamentales (Madrid: Alianza, 2014) 126-136.

3. Es decir que las primeras ideas sobre el control social tienen como punto de partida un enfoque "consensual" de la sociedad. Mathieu Deflem, "Deviance and Social Control", The Handbook of Deviance, ed. Erich Goode (Malden:Wiley Backwell, 2015) 30-44.

4. El mencionado artículo de Deflem, de reciente aparición, se refiere a la historia del concepto en términos más o menos similares a Spierenburg, Janowitz y Melossi.Véase Deflem, "Deviance" 30-44. 
bienestar y la seguridad de la mayoría sin poner en peligro la tranquilidad social". 5 Por su parte, Janowtiz menciona que el concepto fue utilizado desde los inicios de la sociología estadounidense, durante la década 1890, para analizar la organización social y el surgimiento de la sociedad industrial. La perspectiva del control social ayudaba a entender la organización social al enfocarse en los resultados de los mecanismos regulativos. ${ }^{6}$ Melossi plantea que el concepto nació como respuesta a las necesidades de una sociedad en proceso de modernización, industrialización y aparición de nuevos conflictos entre capital y trabajo, en la búsqueda de una respuesta "al reto de mantener la cohesión social dentro de la democracia". " La unidad social, a diferencia de Europa, no era entendida por los estadounidenses en términos políticos sino sociales "como el resultado de un trabajo: la producción de unidad, expuesta a riesgos y al azar”, por lo que no era materia de la filosofia política o de la jurisprudencia, sino de las ciencias sociales. Los sociólogos serían los encargados de "estructurar el marco conceptual de la sociedad estadounidense".

\subsection{El control social como medio de conservación y constitución del orden social}

Edward Ross fue el primer sociólogo en hacer uso explícito del concepto en su libro Social Control. A Survey on the Foundations of Social Order (1901), cuya idea general es que sin control social no hay orden social. ${ }^{9}$ El primero cumple la función de conservar o constituir al segundo: conservar, porque ciertas formas de control basadas en la costumbre se refieren al pasado; constituir, porque otras formas de control provenientes del Estado pueden prever "peligros" no percibidos por la gente y, por tanto, controlarlos por adelantado para producir el orden. Según Ross, el orden social descansa en la idea de que los miembros de un grupo ordenado no "se desvían de su camino agrediéndose entre sí", lo que implica la aceptación de unas normas comunes. ${ }^{10}$ A su juicio, una organización social desarrollada "presupone necesariamente alguna clase de control, puesto que debe existir una autoridad reconocida que separe la línea de demarcación entre los intereses en conflicto". Sin embargo, continúa, "cuando hay cambio y progreso, esas líneas se

5. Peter Spierenburg, "Social Control and History", Social Control in Europe, 1500-1800, vol. 1, eds. Herman Roodenburg y Pieter Spierenburg (Ohio: The Ohio University Press, 2004) 5. Traducción del autor.

6. Morris Janowitz, "Sociological Theory and Social Control", American Journal of Sociology 81.1 (1975): 82-108.

7. Dario Melossi, El estado del control social. Un estudio sociológico de los conceptos de estado y control social en la conformación de la democracia (México: Siglo XXI, 1992) 137.

8. Melossi 145, 150 .

9. Otra de las formas de conservar el orden social es la influencia social. Según Ross, este último concepto se ocupa de la dominación sin intención o propósito, mientras que el control social se refiere a la dominación que tiene un fin y que cumple una función en la sociedad.

10. Aquí aparece claramente el punto de vista consensual de la obra de Ross. Edward Alsworth Ross, Social Control. A Survey on the Foundations of Social Order (New York: The Macmillan Company, 1901) 2. 
hacen inseguras, y si no hay ninguna autoridad para volverlas a dibujar, la sociedad se disuelve en caos". "Aquí aparece la necesidad del control social.

Ross considera que el control social se deriva del "poder social", el cual, a su vez, "es concentrado o difuso en proporción a si los hombres se sienten o no en necesidad de guía o protección". ${ }^{2}$ Es decir, el control social puede estar atribuido a diferentes instancias de la sociedad. Este es el tema de la segunda parte de su libro, sobre los "medios de control" que clasifica en éticos y políticos. ${ }^{13}$ Los primeros, más leves, buscan proteger no solo el orden social, sino un "orden moral" vinculado a él, e incluyen la "opinión pública", la "sugestión", el "ideal personal", la "religión social", el "arte" y la "valoración social". La preferencia por estos medios está directamente relacionada con la homogeneidad del grupo. En cuanto a los medios políticos, más restrictivos, implican la búsqueda de un fin e incluyen la "ley", la "ceremonia", la "educación" y la "ilusión". La preferencia por estos medios se deriva de la heterogeneidad del grupo. ${ }^{14}$ A pesar de esta clasificación, en la obra de Ross es evidente la idea de que las normas de la sociedad, "morales" y "legales", son y deben ser aceptadas por todos.

La postura "monista" de Ross, que presuponía una única sociedad dominante a la que debían adaptarse los inmigrantes, fue dejada de lado por los sociólogos de la Escuela de Chicago (décadas de 1920 y 1930). Para esta, la idea de comunicación, entendida como la ampliación del campo del discurso a través de la democracia, ayudaba a resolver los problemas derivados de las diferencias en la sociedad, entre los que se destacaba la integración de los recién llegados sin necesidad de acudir a la coacción. El problema del control social era entendido por esta escuela como el de la integración práctica de diferentes vocabularios en el universo lingüístico mayor de la sociedad estadounidense. ${ }^{15}$ De acuerdo con Melossi, el New Deal de Franklin Delano Roosevelt, durante el cual la democracia y la opinión pública fueron los principales medios de cohesión social, favoreció la concepción "democrática" del control social de George Herbert Mead (1863-1931) y de John Dewey (1859-1952). Estos autores rechazaban la idea de un punto de vista privilegiado desde donde emanase el control social", sino que por el contrario lo entendían en función de la interacción social en la cual el yo y el control social son dos caras del mismo proceso. ${ }^{16}$

\subsection{El control social como generador del vínculo social}

George Herbert Mead fue el miembro de la Escuela de Chicago que desarrolló con mayor profundidad el concepto. Una de sus preocupaciones era el problema

11. Ross 40. Traducción del autor.

12. Ross 100. Traducción del autor.

13. Ross 89-375.

14. Ross 411.

15. Melossi 156.

16. Melossi 162. 
del orden en una sociedad en permanente cambio. ${ }^{17}$ Sin embargo, en lugar de determinar los diferentes medios externos al individuo en que puede ejercerse el control social, Mead encuentra que es el propio individuo quien ejerce el control social sobre sí mismo.

Mead señala, asimismo, que existe una forma de control históricamente anterior al control social, cuyo funcionamiento es diferente. Esta forma previa de control se dirige a conservar el orden existente; ${ }^{18}$ es externa, basada en el "hábito ciego" y siempre aparece como una obligación: "la ley debe ser obedecida; la constitución debe ser honrada, las diferentes instituciones como la familia, escuela, cortes deben ser reconocidas y obedecidas; el orden que ha llegado a nosotros debe ser preservado". ${ }^{19}$ Toda perturbación debe tener como respuesta el regreso a ese orden, que no puede descomponerse por ser la base de la conducta entre quienes participan en él. ${ }^{20}$

El control social funciona de otra manera. Mead afirma que la Revolución Francesa introdujo el "principio de revolución" en el orden social, lo que dio origen al problema del orden en una sociedad cambiante.Aquí el control no se refiere a la conservación de un orden existente, sino a la generación de un vínculo entre cada miembro del grupo y los demás, del que surgen los individuos capaces de ver reflejado el grupo en ellos y a ellos en el grupo. ${ }^{21}$ El proceso social resulta así el del propio individuo, que adquiere la capacidad de autogobernarse con referencia a sus relaciones tanto con el grupo en general como con los otros individuos. El control social es así tanto autocrítica como crítica social, "y la conducta controlada por la autocrítica es conducta controlada socialmente". Por ello, según Mead, el control social no elimina al individuo y su individualidad, sino que lo constituye. El control y el individuo están inevitablemente unidos, porque "este es lo que es, en cuanto personalidad consciente e individual, en la medida en que sea un miembro de la sociedad, involucrado en el proceso social de la experiencia y la actividad, y, por lo tanto, socialmente controlado en su conducta". ${ }^{22}$ El control, además de constituir a los individuos, les da su carácter como miembros de un grupo. ${ }^{23}$

17. Anselm Strauss, "Introduction", The Social Psychology of George Herbert Mead, ed. Anslem Straus (Chicago: The University of Chicago Press, 1956) ix.

18. George Herbert Mead, "The Industrial Revolution", Movements of Thought in the Nineteenth Century, ed. Merritt H. Moore (Chicago: University of Chicago Press, 1936) 176-177.

19. George Herbert Mead,"The Problem of Society - How we become Selves", The Social Psychology of George Herbert Mead, ed. Anslem Straus (Chicago:The University of Chicago Press, 1956) 18. Traducción del autor.

20. Mead, "The Problem of Society" 18.

21. George Herbert Mead, "The Genesis of the Self and Social Control”, International Journal of Ethics 35.3 (1925): 274.

22. George Herbert Mead, Espíritu, persona y sociedad. Desde el punto de vista del conductismo social (Buenos Aires: Paidós 1972) 271

23. Mead, Espíritu 280. 
Mead comparte con Ross una idea consensual de la sociedad, sin embargo, la relación entre control y orden social es diferente en ambos autores. Para Mead en las comunidades el orden es garantizado por el control no por el acuerdo, mientras que en la sociedad el orden se deriva del control social entendido como una relación entre individuos no como conjunto de medios de control. Asimismo, si en Ross el control social puede conservar o servir de fundamento para el orden social, en Mead lo constituye, pero de una forma en la que de todos modos se presume la generación de un vínculo aceptado por todos los individuos. Por ello, para Mead el medio de resolver los conflictos en la sociedad no es el control social, sino la reconstrucción social. Esto significa que en una sociedad altamente desarrollada y organizada los conflictos son resueltos mediante "reconstrucciones de las situaciones sociales particulares", lo que "presupone una base de intereses sociales comunes compartidos por los miembros individuales de la sociedad humana dada en la que ocurre dicha reconstrucción". ${ }^{24}$ Es evidente que dichos "intereses compartidos" se derivan del vínculo generado entre los individuos, gracias al control social tal como lo entiende Mead, y que la reconstrucción social es un proceso posterior.

Las definiciones de Ross y Mead forman parte de las definiciones clásicas del control social. En ellas, como destaca Morris Janowitz, el concepto se refería a la capacidad de un grupo para regularse a sí mismo en términos de principios morales legítimos. Janowitz también señala que, si el concepto es entendido de esta manera, se diferenciaría entonces del control coercitivo, de la organización de la sociedad basada en la amenaza o uso de la fuerza. Sin embargo, como indica el mismo Janowitz, una definición más precisa del control social comenzó a aparecer desde la década de 1940, cuando el concepto se asimiló tanto a la socialización como a la coerción. ${ }^{25}$

\subsection{El control social como función de respuesta a la desviación}

Melossi coincide con Janowitz en que el concepto se transformó y se limitó al enfocarse ante todo en el problema de la desviación y del crimen. Según Melossi, de un control social activo, basado en la producción de comportamientos, el concepto pasó a ser ante todo reactivo; es decir, dirigido a reprimir o prevenir las conductas desviadas. De acuerdo con Melossi, esta transformación estaba vinculada a situaciones como la Gran Depresión y el ascenso al poder de regímenes fascistas y comunistas que amenazaban la cohesión social. Esto implicó una reorientación en el vocabulario político y social en obras como Social Control Through Law (1942) de Roscoe Pound (1870-1964), que destacaban la importancia de la certidumbre

24. George Herbert Mead, "Society", The Social Psychology of George Herbert Mead, ed. Anslem Straus (Chicago:The University of Chicago Press, 1956) 281. Traducción del autor. En este punto se hace evidente la influencia de la obra de Durkheim, particularmente La división del trabajo social (1893).

25. Janowitz muestra con ejemplos que las nuevas formas de entender el concepto no desplazaron del todo la perspectiva tradicional, la cual siguió apareciendo en algunos trabajos sociológicos de la segunda mitad del siglo XX. Janowitz 82-108. 
de la ley para el control social. ${ }^{26}$ Asimismo, en la década de 1950 apareció una obra clave para entender esta transformación del concepto: The Social System (1951) de Talcott Parsons (1902-1979), en la que define el control social como la respuesta a la desviación. Así, para este sociólogo el control social es la función de responder a la desviación para conseguir la conformidad de los individuos al sistema social. La conformidad es la adecuación de los individuos a la estructura establecida y a los requerimientos funcionales del sistema social, mientras que la desviación se refiere a las perturbaciones en el equilibrio del sistema, que específicamente implican contravenciones de los patrones institucionalizados que son el punto focal del sistema social.

Según Parsons, estos patrones institucionalizados constituyen la "guía de la conducta y de las relaciones sociales que se han entretejido con un sistema común de sentimientos morales que a su vez definen lo que uno tiene 'derecho de esperar' de una persona en una determinada posición". ${ }^{27}$ Dichas definiciones normativas de la conducta propia y de lo que se puede esperar de la ajena están estructuradas en una dimensión de conformidad-desviación: en la interacción social los individuos "premian" la conformidad de otros individuos, mientras que "castigan" su desviación. Este primer mecanismo de control es informal, es "privado" y espontáneo, basado en un sistema de sentimientos morales, y reacciona favorablemente a la conformidad y desfavorablemente a la desviación de los patrones institucionalizados dentro de los que se desenvuelve la acción social. A su vez, el sistema formal de sanciones no podría funcionar a menos que esté soportado por dicho sistema informal. ${ }^{28}$

De acuerdo con Parsons, estos mecanismos de control social existen desde el comienzo de la socialización y permanecen a lo largo de la vida del individuo. ${ }^{29} \mathrm{El}$ proceso de socialización se refiere a la adquisición de las orientaciones necesarias para la acción dentro de los patrones institucionalizados; es un proceso previo al control social, pero se dirige a lo mismo: conservar el funcionamiento "normal" del sistema social.

El sistema social de Parsons es ante todo una abstracción con fines analíticos, pero tiene también connotaciones normativas. Es una idea sobre un orden social cuyo funcionamiento implica la ausencia de contradicciones entre las normas legales y los "sentimientos morales" de los individuos, o en general de las normas consuetudinarias que puedan existir en la realidad. Por ello, de acuerdo con Parsons, los medios formales e informales de control cumplen la misma función, y los segundos no podrían oponerse a los primeros sin convertirse en desviaciones.

26. Roscoe Pound, Social Control Through Law (New Brunswick: Transaction Publishers, 1997).

27. Talcott Parsons, Essays in Sociological Theory (New York:The Free Press, 1954) 143. Traducción del autor.

28. Talcott Parsons, The Social System (London: Routledge, 1991).

29. Parsons, The Social 91. 
Los planteamientos del funcionalismo sociológico sobre control social, conformidad y desviación aparecen en la criminología estadounidense dentro de la teoría del control. El mismo año de la aparición de The Social System fue publicado el artículo "Delinquency as the Failure of Personal and Social Controls" de Albert J. Reiss (1922-2006), en el que dichos términos están enfocados en el problema de la delincuencia. ${ }^{30}$ Según Reiss, esta sería un resultado del fallo de los controles personales y sociales para producir comportamientos conformes con las normas del sistema social al que están ligadas las penas legales. ${ }^{31}$ La teoría criminológica del control continuó desarrollándose en la década de 1960 por autores como Travis Hirschi (1935) con un enfoque aún más psicologizante y prescriptivo. ${ }^{32}$

En este punto podría ser útil aclarar la distinción entre medios formales e informales de control en la sociología estadounidense, aunque su sentido probablemente ya ha sido comprendido por el lector. El control formal, llamado a veces legal, es el que ejercen funcionarios especializados, socialmente delegados para la tarea de controlar un grupo. El control informal no se delega en una unidad especial, sino que lo ejercen todos los miembros del grupo como unidad. ${ }^{33}$ Es de destacar que lo anterior coincide nuevamente con las definiciones de Weber sobre las garantías de la legitimidad de un orden convencional y de derecho. ${ }^{34}$

A pesar de la apropiación criminológica del concepto, el control social continuó apareciendo en obras sociológicas de mayor alcance. El ya mencionado Morris Janowtiz, por ejemplo, propone entender el control social como una perspectiva enfocada en la capacidad de autorregulación de una organización

30. Albert J. Reiss, "Delinquency as the Failure of Personal and Social Controls", American Sociological Review 16.2 (1951): 196-207.

31. Véase Michael David Wiatrowski, “Social Control Theory and Delinquency” (Tesis de doctorado en Estudios Urbanos, Portland State University, 1978); Lara Helena Kuhn, Social Control and Human Nature: What Is It We Are Controlling? (El Paso: LFB Scholarly Publishing LLC, 2009). Dentro de la teoría del control tuvo lugar un giro que profundiza en los aspectos individuales, psicológicos, en la conceptualización sobre el crimen, con la obra A General Theory of Crime (1990) de Travis Hirschi (1935) y Michael Gottfredson (1951). Los autores dan prelación al autocontrol sobre el control social para comprender el delito que sería consecuencia de la falta de autocontrol individual. Kuhn 25-26. Asimismo, Travis Hirschi, "In defense of Self-Control", Theoretical Criminology 4.1 (2000): 5-69.

32. Véase Travis C. Pratt y otros, "Key Idea: Hirschi’s Social Bond/Social Control Theory”, Key Ideas in Criminology and Criminal Justice (New York: SAGE, 2010) 56-57; Travis Hirschi and David Rudisill, "The Great American Search: Causes of Crime, 1876-1976", Annals of the American Academy of Political and Social Science 423 (1976): 14-22.

33. Citado en John Griffiths "The Division of Labor in Social Control", Toward a General Theory of Social Control: Selected Problems, vol. 2, ed. Donald Black (Orlando: Academic Press, 1984) 39-40.

34. Según Max Weber, en un orden de derecho, la legitimidad está garantizada por un aparato de personas que pueden usar "la coacción física o psíquica, para cumplir el orden y para perseguir las transgresiones". A su vez, en un orden de tipo convencional, dentro de un grupo humano, la garantía está en "la probabilidad de que, en caso de desviación, se encontrará con una desaprobación relativamente general y perceptible". Weber 131-132. 
social. ${ }^{35}$ Asimismo, Mathieu Deflem (1962) señala que desde la década de 1980 aparecieron nuevas "perspectivas revisionistas" sobre el control social en las que este se estudiaba sociológicamente como un tema autónomo no necesariamente vinculado al delito. Según Deflem, estos trabajos se enfocan en "las dimensiones sociales y sociológicas relevantes de los agentes, instituciones y mecanismos de control social, independientemente de su rol en el crimen o desviación. En este sentido, los representantes de este nuevo movimiento han pasado de una criminología explícita o implícita a una sociología en estricto sentido". ${ }^{36}$

La teoría del etiquetaje de Howard Becker (1928), desarrollada en la década de 1970, puede entenderse como una posición revisionista ante el control social, no como concepto (que recoge de las definiciones clásica y funcionalista), sino como práctica social contra los individuos. Para Becker, la desviación implica la calificación negativa de la conducta individual por parte del grupo a través de los "controles sociales", que operan precisamente para "mantener las formas aceptadas de conducta". Estos controles, según Becker, afectan la conducta individual a "través del uso de poder y la aplicación de sanciones. La conducta aceptada es premiada y la conducta valorada negativamente es castigada. El control sería difícil de mantener si la fuerza fuera siempre necesaria, por lo que surgen mecanismos que realizan la misma función". ${ }^{37}$ La obra de Becker, por tanto, sería en el fondo una crítica a la existencia de los controles informales antes indicados.

Vale la pena destacar también la obra de Stanley Cohen (1942-2013) Visions of Social Control (1985), sobre todo por dos razones: su contenido histórico y su posición crítica ante el control social, que no solo coincide con la obra de Becker (centrada, a diferencia de la suya, en los controles informales), sino que, además, es la que tienden a compartir los historiadores que se inspiran en la obra de Michel Foucault. Cohen considera que el control social se refiere a las respuestas a la desviación para conseguir conformidad. ${ }^{38}$ Con esto parece recoger la definición funcionalista, aunque su interpretación, influida por Foucault, es diferente. Según Cohen, las formas en las que en los Estados occidentales se ha enfrentado a la desviación se transformaron desde finales del siglo XVIII y conformaron una red de control mucho más extendida en la segunda mitad del siglo XX. El crecimiento de la red, de instituciones y de profesionales en materia de control está en relación directa con la incorporación de nuevas formas de desviación. Aunque Cohen no define explícitamente la conformidad, esta aparece con unas connotaciones autoritarias ajenas a la definición funcionalista.

35. Janowitz 84.

36. Deflem, "Deviance" 19. Traducción del autor.

37. Howard Becker, Outsiders. Studies in the Sociology of Deviance (New York:The Free Press, 1966) 59 y 60. Traducción del autor. Esta obra contrasta particularmente con la antes mencionada teoría criminológica del control surgida en ese mismo período, al enfatizar ya no en las formas en que debe controlarse la desviación, sino en la producción social de aquella.

38. Stanley Cohen, Visions of Social Control. Crime, Punishment and Classification (Cambridge: Polity, 1985) 2-4. 


\subsection{El control social como resolución de conflictos}

La teoría de Donald Black, desarrollada sobre todo en The Social Structure of Right and Wrong (1993), asimila el control social a la resolución de conflictos. Su definición de control social parece derivada del funcionalismo: se trata de "las formas en que la gente define y responde a la conducta desviada"; 39 sin embargo, Black señala que su teoría "no involucra presunciones o implicaciones concernientes al impacto del control social sobre la conformidad, orden social o cualquier otra cosa, ni se refiere a los significados subjetivos del control social para aquellos que lo ejercen o experimentan". ${ }^{40}$ No le interesa la influencia que puedan tener los mecanismos de control social, llámese conformidad o de otra forma, sino sus manifestaciones a través de las que se expresan los estándares sobre lo correcto y lo incorrecto en un grupo. De este modo, el control social en la obra de Black consiste en las formas en que se expresan los agravios y el manejo de los conflictos derivados de ellos. Estas formas pueden ser tantas según el carácter normativo del grupo y la forma del agravio: el control social comprende desde un gesto de desaprobación hasta el bombardeo a una ciudad, por ejemplo.

La teoría de Black permite incluir algunas formas de violencia dentro de los mecanismos de control social. Los linchamientos, asesinatos por honor, torturas, duelos o destrucción de propiedades no serían siempre desviaciones, sino más bien manifestaciones de los aspectos conflictivos de un orden social que dan cuenta, al mismo tiempo, de la legitimidad de ciertas formas violentas de resolución de conflictos no monopolizadas por el Estado. ${ }^{41}$ Además, la localización de estas "violencias legítimas" en el espacio social destacaría la relación entre ellas y la ausencia del Estado o la "distribución" desigual de la ley —el medio "estatal" de control social— en una sociedad. ${ }^{42}$ De hecho, Black señala que la forma básica del control social, propia de "sociedades campesinas y tribales", "autoayuda" (self-help) y sigue funcionando en sociedades modernas en relación inversa a la posibilidad de acceso a los mecanismos legales. Por ello, Black acepta que ciertas conductas definidas como crímenes por el Estado constituyan formas de control social, lo cual contradice la presunción funcionalista de identificación entre los controles formal e informal.

39. Donald Black, The Social Structure of Right and Wrong (San Diego: Academic Press, 1997) 2. Traducción del autor.

40. Donald Black, "Crime as Social Control”, American Sociological Review 48.1 (1983): 34-45. Traducción del autor. Este artículo, en una versión extendida, forma parte del libro antes mencionado.

41. Por ejemplo, en algunos estados de Estados Unidos, hasta la primera mitad del siglo XX era común el linchamiento de negros o mexicanos.

42. Para Black debería ser aparente la no existencia de un monopolio absoluto de la fuerza por parte del Estado, ni siquiera en Estados Unidos. Black, The Social 36. 


\subsection{El control social como forma de poder}

Como se señaló, la obra de Foucault está vinculada a las concepciones revisionistas sobre el control social y se trata de una influencia importante en la historiografia. La principal diferencia entre las aproximaciones clásicas y funcionalistas y la obra de Foucault es que para este el control social es ante todo una manifestación del poder. De hecho, para Foucault toda relación social es de poder, de lo que puede concluirse que sus ideas sobre el poder se tratan realmente de un presupuesto sobre las relaciones sociales. ${ }^{43}$ En el modelo de poder del primer tomo de su Historia de la sexualidad, el elemento de coacción de la relación social se encuentra solo en uno de sus extremos, mientras los otros carecen por completo de él. Foucault lo denomina fuerza, puesto que le interesa destacar que va más allá de la restricción: la parte que ejerce el poder está en posición no solo de prohibir, obligar o imponer, sino incluso modificar a la otra parte y determinar sus acciones. Por otro lado, su ejercicio implica la resistencia, es decir, la oposición de la otra parte de la relación. ${ }^{44}$ En síntesis, para Foucault el poder siempre está distribuido de una forma absolutamente desequilibrada.

Si toda relación social es de poder distribuido de forma absolutamente desigual, siguiendo a Foucault, todo orden social estaría constituido exclusivamente por relaciones absolutamente desiguales de poder. Y si el ejercicio del poder implica resistencia por parte de quienes no lo ejercen, podría concluirse que todo orden social es en principio ilegítimo para quienes no están en la primera posición y que el poder sería el fundamento de la aceptación o legitimidad de las normas del orden social.

Foucault se interesa sobre todo en el poder de normalización que tiende a la homogeneización, clasificación y organización de los sujetos en función de los intereses de producción y de conocimiento del grupo dominante en la sociedad moderna: la burguesía. La normalización adscribe a los sujetos a un "cuerpo social homogéneo", lo que implica que la legitimidad del orden social moderno se deriva del poder de normalización. ${ }^{45}$ Este poder se ejerce en forma de control sobre los cuerpos — por lo que Foucault lo llama también disciplinario-, para cuyo

43. Foucault menciona el "carácter estrictamente relacional de las relaciones de poder" y "que las relaciones de poder no están en posición de exterioridad respecto de otros tipos de relaciones [...] sino que son inmanentes". Michel Foucault, Historia de la sexualidad, vol. 1 (México: Siglo XXI, 1991) 67.

44. Según Foucault, "donde hay poder hay resistencia, y no obstante (o mejor: por lo mismo), ésta nunca está en posición de exterioridad respecto del poder”. Foucault, Historia 117.

45. "En un sentido, el poder de normalización obliga a la homogeneidad; pero individualiza al permitir las desviaciones, determinar los niveles, fijar las especialidades y hacer útiles las diferencias ajustando unas a otras. Se comprende que el poder de la norma funcione fácilmente en el interior de un sistema de la igualdad formal, ya que en el interior de una homogeneidad que es la regla, introduce, como un imperativo útil y el resultado de una medida, todo el desvanecido de las diferencias individuales". Michel Foucault, Vigilar y castigar. Nacimiento de la prisión (Buenos Aires: Siglo XXI, 2002) 190. 
ejercicio surgió un conjunto de instituciones y prácticas dirigidas a controlar los cuerpos y docilitarlos con el fin de volverlos útiles y obedientes. A esta relación docilidadutilidad Foucault la llama disciplina, que "aumenta las fuerzas del cuerpo (en términos económicos de utilidad) y disminuye esas mismas fuerzas (en términos políticos de obediencia). ${ }^{46}$ En su obra Vigilar y Castigar, Foucault menciona diferentes formas en las que se pone en práctica este control, enfocándose en la prisión. ${ }^{47}$

La teoría de Foucault estimuló los estudios históricos sobre las instituciones de control al ponerlas como foco de una transformación histórica importante. Talcott Parsons ya había considerado la importancia del cuerpo físico como objeto de control, por ejemplo, al incluir el retraimiento derivado de la enfermedad como una forma de desviación y a la práctica médica como un medio para controlarla. ${ }^{48}$ Sin embargo, su obra carece de la dimensión histórica de la Foucault, y no tuvo la influencia del francés. Después de Foucault la historiografia sobre cada una de las instituciones de control es un área tan extensa que sería necesario dedicar un espacio para cada una de ellas.

\section{Usos del concepto de control social en la historiografía}

En esta sección se analizarán algunos ejemplos del uso del concepto de control en investigaciones históricas. Los artículos fueron escogidos entre la historiografia estadounidense para mostrar, por un lado, su apropiación temprana por los historiadores y, por otro, la claridad con que utilizan el concepto en sus análisis sobre órdenes sociales del pasado. De ese modo, se pretende contribuir a la comprensión del uso del concepto en casos concretos. Los ejemplos escogidos muestran, asimismo, que el uso historiográfico del control social tiene un mayor alcance sociológico que el de "analizar nuevos sujetos sociales y tópicos hasta entonces inéditos [...] como los marginados sociales, los delincuentes, la protesta social [...] y en análisis de género", como se concluye en un balance historiográfico sobre el tema. ${ }^{49} \mathrm{De}$ todos modos, lo anterior parece, en términos generales, una tendencia importante en la historiografia latinoamericana. ${ }^{50}$

Sin hacerlo explícito, algunos de los trabajos históricos revisados usan el concepto de una forma más o menos similar a la definición clásica; otros se interesan por un medio particular de control, formal o informal, o examinan la relación

46. Foucault, Vigilar 143.

47. Como la fábrica, la escuela y el asilo. Foucault, Vigilar 177.

48. Parsons, The Social 19 y 288-322. La mencionada similitud ameritaría una comparación más detallada entre las obras de estos autores.

49. Juan José Marín Hernández, "El control social y la disciplina histórica. Un balance teórico metodológico", Revista de Historia de América 129 (2001): 70.

50. Véase, por ejemplo, Eugenia Bridikhina, Sin temor a Dios, ni a la justicia real: control social en Charcas a fines del siglo XVIII (La Paz: Instituto de Estudios Bolivarianos / Facultad de Humanidades y Ciencias de la Educación, 2001); Ivonne Bravo Páez, Comportamientos ilícitos y mecanismos de control social en el Bolivar Grande, 1886-1905 (Bogotá: Ministerio de Cultura, 2002). 
entre los medios formales o informales. También es posible encontrar trabajos que adoptan un enfoque revisionista o crítico del control social influidos por la obra de Foucault.

Una primera forma de usar el concepto, derivada de la concepción original de Ross, es la identificación de los diferentes medios de control social con el fin de aproximarse a un orden social en el pasado. Sylvia L. Thrupp (1903-1997) hace esto en su artículo de 1941 sobre los pueblos medievales, al parecer el primer texto historiográfico en apropiarse del concepto. Para la autora, el control social estaba encaminado "a formular los fines de la acción y a controlar la conducta", y muestra que era llevado a cabo por diversos "núcleos de autoridad" que funcionaban como "agencias de control" en la comunidad urbana: la Iglesia, la autoridad del rey o del señor feudal, los gremios, la costumbre y la familia. Además de los medios de control ubicados en una instancia determinada, la autora se refiere al medio más disperso de la opinión pública, que incluye desde el chisme en la parroquia hasta las ideas de los "reformadores y satíricos". ${ }^{51}$ La autora parece presuponer la legitimidad generalizada del orden social, garantizada tanto por las autoridades como por la población.

Hay investigaciones que especifican cómo el control social se dirige a proteger un orden dominante; por ejemplo, el artículo sobre Massachusetts de David H. Flaherty. ${ }^{52}$ El autor examina el sistema de control social contra el crimen, dirigido a conservar el orden social durante los siglos XVI y XVII, e identifica los medios formales e informales de control en pueblos. Los medios formales incluían a las audiencias donde se juzgaban los delitos y a los "oficiales de la ley" como los jueces de paz, los Sheriffs, los diputados y los alguaciles. Entre los medios informales estaban las élites regionales, que funcionaban como instancias de control al dominar la vida política y judicial, la Iglesia, la propia vigilancia de la comunidad, además de la familia, cuyo rol en la socialización y en la internalización de las normas era más importante en este período que en la era industrial, según Flaherty. Por otra parte, la servidumbre era una de las formas de controlar a los negros e indios, quienes eran considerados como desviados por los miembros de la sociedad dominante, es decir, por fuera de su orden social. El control social se encaminaba a proteger ese orden dominante, caracterizado según Flaherty por la homogeneidad y respeto por la ley entre quienes participaban de él.

Un artículo con un enfoque similar, pero con unas conclusiones de mayor alcance, trata sobre el crimen en Estados Unidos durante el período colonial. Luego de comparar los sistemas de justicia y control social de diferentes provincias, Douglas Greenberg encuentra una relación directa entre la supresión efectiva del crimen a través del control y la imposición de un orden social jerárquico y autoritario. La delincuencia era menor en las colonias caracterizadas por la represión

51. Sylvia L. Thrupp, "Social Control in the Medieval Town”, The Tasks of Economic History 1 (1941): 42.

52. David H. Flaherty, "Crime and Social Control in Provincial Massachusetts", The Historical Journal 24 (1981): 339-360. 
exitosa del disenso y de las críticas a la élite dominante, mientras que en las colonias de mayor heterogeneidad étnica y religiosa, de "mayor actividad política democrática", había mayores dificultades para lidiar con el crimen y con la falta de deferencia al sistema legal. Para el autor, "donde floreció el orden en la América colonial, también lo hizo la opresión; donde prosperó la libertad, también lo hizo el crimen". 53

Existen trabajos que estudian los medios informales de control en su función de conservar un orden social dominante. Por ejemplo, el artículo de Clifford S. Griffin, referido al protestantismo evangélico durante un período de cambios radicales en la sociedad estadounidense como la industrialización, la inmigración y el aumento de la población. Las sociedades de beneficencia del protestantismo evangélico fueron una respuesta en forma de control social a dichos cambios, dirigida a dictar la conducta de los grupos que estaban surgiendo y a establecer unos estándares sobre lo correcto en la sociedad. ${ }^{54}$ Es evidente que en este artículo está presente la definición clásica del control social, pero con unas connotaciones críticas que llaman la atención, si se tiene en cuenta el año de su aparición (1957), y que se evidencian sobre todo cuando el autor vincula las sociedades de beneficencia al partido Republicano y a su posición en contra de la diferencia y el disenso. ${ }^{55}$

El tema de la opinión pública como medio de control social informal también ha sido desarrollado. Un ejemplo, en este caso inglés, es el artículo de Elizabeth Foyster sobre honor masculino y golpizas a esposas en Inglaterra durante el siglo XVII. ${ }^{56}$ Según la autora, las golpizas eran una forma de control social en un orden patriarcal en el que el honor público de los hombres dependía del que recibían en sus casas. Sin embargo, Foyster también da cuenta de una desaprobación pública a las golpizas excesivas, puesto que se tratarían de acciones ajenas a la razón, una característica considerada exclusivamente masculina. Esta segunda idea está dando cuenta más bien de la opinión pública como medio de control social por lo cual la autora se equivoca al incluir dentro de este solo al maltrato, sobre todo teniendo en cuenta la conclusión más importante que resulta del artículo: el orden social patriarcal descansaba más en ideas generalizadas sobre las diferencias entre los sexos que en la imposición física de uno de ellos sobre el otro.

53. Douglas Greenberg, "Crime, Law Enforcement, and Social Control in Colonial America", The American Journal of Legal History 26.4 (1982): 325. Traducción del autor.

54. Clifford S. Griffin, "Religious Benevolence as Social Control, 1815-1860: The Mississippi Valley", Historical Review 44.3 (1957): 423-444.

55. Este artículo fue criticado por Lois W. Banner, quien ofrece una relación diferente entre las sociedades de beneficencia y el orden social en Estados Unidos, además de criticar el uso del concepto de control social. Para esta autora, las organizaciones de beneficencia no buscaban "controlar" la sociedad o conservar un orden religioso en la sociedad, sino que pretendían contribuir al "éxito" de la república americana para alcanzar un orden democrático a través de la reforma. Lois W. Banner, "Religious Benevolence as Social Control: A Critique of an Interpretation", The Journal of American History 60.1 (1973): 23-41.

56. Elizabeth Foyster, "Male Honour, Social Control and Wife Beating in Late Stuart England", Transactions of the Royal Historical Society 6 (1996): 215-224. 
Por otra parte, es posible encontrar investigaciones que problematizan la relación entre los medios de control social formales e informales. Dos de estos casos se refieren a la violencia. En primer lugar, un artículo de Charles David Phillips en el que se destaca su crítica de la teoría de Donald Black a partir de una perspectiva del conflicto. ${ }^{57}$ Phillips muestra que entre 1889 y 1918 en North Carolina puede identificarse una relación directa entre la cantidad de linchamientos a personas de color y sus ejecuciones por decisión judicial. Con esto contradice los planteamientos de Black, quien estima que existe una relación inversa entre la aplicación de medios informales y la de los medios formales de control. Según Phillips es necesario considerar las características del conflicto social en las que dichos eventos funcionan como instrumentos de control. El autor muestra que en períodos de mayor conflicto racial se incrementaban tanto las ejecuciones como los linchamientos, cumpliendo ambos el mismo propósito de conservar la dominación blanca.

En segundo lugar, otro artículo sobre linchamientos en el sur de Estados Unidos, a diferencia del anterior, confirma una relación inversa entre los medios violentos formales de control y los informales. ${ }^{58}$ Los autores reconocen que la obra de Black no se refiere a las dinámicas culturales detrás de los medios informales de control, aunque destacan la importancia que dicho sociólogo da a la falta de acceso a la ley y su relación con la "autoayuda". El artículo muestra cómo los linchamientos contribuyen a la reproducción de la violencia durante el siglo XX: por un lado, el "efecto brutalizador" de los linchamientos se reproduce en el aumento de ejecuciones judiciales a personas de color al ir disminuyendo aquellos durante el siglo XX. Por otra parte, las adaptaciones de los negros a una situación en la que la ley tendía a actuar en su contra los fue llevando a generalizar el uso de la autoayuda como medio de control social en lugar de acudir a mecanismos legales.

Asimismo, el control social también ha sido visto históricamente como una ideología derivada de los intereses de un grupo que alcanza una posición dominante. ${ }^{59}$ Por ejemplo, William Issel desde un enfoque marxista señala que en Pennsylvania la escuela sirvió para reproducir las ideologías "del control social, de la americanización y la aculturación" en respuesta a las amenazas del conflicto de clases y de la diversidad étnica, en un período de cambios relacionados con el capitalismo industrial, y en función de los intereses de los administradores de las escuelas, quienes estaban surgiendo como grupo al redefinir su rol en respuesta al capitalismo como agentes profesionales.

Es posible encontrar también obras que se enfocan en los mecanismos formales de control social para materializar unas ideas sobre el orden social. Es, por ejemplo,

57. Charles David Phillips, "Exploring Relations among Forms of Social Control:The Lynching and Execution of Blacks in North Carolina, 1889-1918”, Law \& Society Review 21.3 (1987):361-374.

58. Steven F. Messner y otros, "The Legacy of Lynching and Southern Homicide", American Sociological Review 70.4 (2005): 633-655.

59. William Issel, “Americanization, Acculturation and Social Control: School Reform Ideology in Industrial Pennsylvania, 1880-1910”, Journal of Social History 12.4 (1979): 569-590. 
el caso del artículo de Mark D. Schuzman sobre la justicia en Buenos Aires, Argentina, después de la Independencia. ${ }^{60}$ Según Schuzman, las nuevas autoridades buscaron establecer y conservar la ley y el orden con ayuda del sistema judicial; sin embargo, las realidades del orden social existente, caracterizado por la prevalencia de intereses privados y locales por encima de criterios normativos abstractos, hicieron imposible su pretensión de crear un Estado según el modelo europeo. Además, la propuesta resulta de interés porque el control social aparece vinculado a un grupo social interesado en modificar el orden legítimo existente a partir de unas ideas nuevas sobre el orden. En este sentido, remite a la idea foucaultiana del control como forma de poder y generador de la legitimidad. Sin embargo, al destacar el fracaso de la implementación de una justicia guiada solo por los criterios generales de las leyes republicanas, evidencia que el nuevo orden promovido desde el Estado no reemplazó al orden social existente, sino que entró en coexistencia con él. Un indicio de ello es el uso que los actores sociales, incluidos los mismos funcionarios, hacían de los mecanismos judiciales en beneficio de sus propios intereses locales.

Un tema escasamente examinado ha sido el del control social más allá de los límites nacionales. De todos modos, existe un ejemplo en el artículo de Mathieu Deflem sobre el surgimiento de la Interpol a mediados del siglo XX. ${ }^{61}$ Deflem se refiere a la internacionalización del control social formal a través de la colaboración de las instituciones policiales, le interesa sobre todo destacar la "independencia burocrática" de dichas organizaciones, cuyas estructuras y mecanismos eran autónomos con respecto a los dictados ideológicos de los Estados, según el autor. Por más discutible que sea el enfoque de Deflem, el tema general que propone es interesante y merece más atención de la que ha tenido. Pienso, por ejemplo, en el papel que podrían tener los medios de comunicación en la producción de ciertas formas de control social informal en un contexto global.

En cuanto a la historiografia latinoamericana, se observa que la crítica al control social como práctica y la influencia de Foucault han sido particularmente importantes, por ejemplo, en temas como la prisión. ${ }^{62}$ Como se mencionó previamente, esta historiografía requeriría de análisis enfocados solo en ella; sin embargo, existen dos obras sobre la prisión en América Latina que vale la pena mencionar. En la primera de ellas, editada por Ricardo Salvatore y Carlos Aguirre, la influencia de Foucault es más notoria. ${ }^{63}$ En este libro el surgimiento de la penitenciaría

60. Mark D. Szuchman, "Disorder and Social Control in Buenos Aires, 1810-1860", The Journal of Interdisciplinary History 15.1 (1984): 83-110.

61. Mathieu Deflem, "Bureaucratization and Social Control: Historical Foundations of International Police Cooperation”, Law \& Society Review 34.3 (2000): 739-778.

62. Existen otras investigaciones que aplican este punto de vista a procesos como las reformas borbónicas o la formación de los Estados nacionales. Por ejemplo, los libros Bridikhina, Sin temor; Bravo, Comportamientos.

63. Ricardo D. Salvatore y Carlos Aguirre, eds., The Birth of the Penitentiary in Latin America. Essays on Criminology, Prison Reform, and Social Control, 1830-1940 (Austin: University of Texas Press, 1996). 
aparece vinculado a los esfuerzos modernizadores de los Estados y en función de los intereses de los grupos dominantes. El texto enfatiza más en estos intereses por controlar a la población a través de la penitenciaría que en la realización efectiva de las ideas que impulsaban la implementación de dicha institución de control. Por su parte, el libro editado por Ernesto Bohoslavsky y Maria Silvia Di Liscia puede verse como una respuesta al de Aguirre y Salvatore, puesto que destaca las dificultades que los grupos dominantes tuvieron para hacer efectivas sus ideas de ordenación de la sociedad a través de las instituciones de control. ${ }^{64}$

\section{Conclusiones}

En primer lugar, una de las principales diferencias entre las distintas teorías sobre el control social es la connotación que le dan: en general, en la sociología clásica tiende a verse como una necesidad social, mientras que en las teorías revisionistas, en las que la sociedad suele ser vista desde una perspectiva conflictiva, el concepto adquiere unas connotaciones más represivas. Un problema derivado de lo anterior puede ser un interés excesivo en los medios formales de control, como en algunos trabajos históricos mencionados. Sin embargo, además de que existen investigaciones que resaltan la importancia de los medios informales de control, es posible ver cómo otros trabajos han llegado a problematizar la relación entre ambas clases de medios.

En segundo lugar, también queda claro que es posible seguir investigando en otras formas de entender el control social u otros conceptos referidos al mismo tipo de problemas. La obra de Norbert Elias, por ejemplo, aparece citada por autores como Donald Black, y dentro de su teoría de la civilización hay planteamientos históricos sobre las transformaciones en las formas de coacción y autocoacción entre individuos que pueden ser de gran importancia e interés para los historiadores interesados en el control más allá de la obra de Foucault. Por otro lado, la obra de Harrison White sobre la relación entre identidad y control puede ser asimismo útil para los historiadores interesados en los problemas relacionados con el orden social. ${ }^{65}$ Quedan otros temas sobre los que valdría la pena profundizar tales como las instituciones de control más allá de la prisión, la relación entre género y control social y los medios informales de control en la historia contemporánea en América Latina. Estos serían solo algunos ejemplos.

También es importante resaltar que en este artículo no se propuso una definición o uso "correctos" del término. Sin embargo, se observa que algunos de sus usos pueden implicar simplificaciones de la realidad en función de unos intereses

64. Maria Silvia Di Liscia y Ernesto Bohoslavsky, Instituciones y formas de control social en América Latina. Una revisión (Buenos Aires: Prometeo, 2005).

65. Harrison C. White, Identity and Control (Princeton: Princeton University Press 2008). Aunque no fue incluida en este artículo al no referirse en estricto sentido al concepto de control social, se enmarca dentro de la misma tradición sociológica estadounidense interesada en la constitución del orden social de varios de los textos revisados. 
o presunciones del historiador como puede ser el considerar al Estado solo como una herramienta de opresión. Lo más importante sería que el investigador tenga una idea lo más clara posible sobre el concepto de control social. En este sentido, el estudio del desarrollo del concepto es un ejercicio importante para darse cuenta de la atención que se debe dedicar a cada uno de los términos que aparecen dentro del análisis histórico.

\section{Bibliografía}

Banner, Lois W. "Religious Benevolence as Social Control: A Critique of an Interpretation". The Journal of American History 60.1 (1973): 23-41.

Becker Howard. Outsiders. Studies in the Sociology of Deviance. New York: The Free Press, 1966.

Black, Donald. The Social Structure of Right and Wrong. San Diego: Academic Press, 1997.

."Crime as Social Control". American Sociological Review 48.1 (1983): 34-45.

Bravo Páez, Ivonne. Comportamientos ilícitos y mecanismos de control social en el Bolívar Grande, 1886-1905. Bogotá: Ministerio de Cultura, 2002.

Bridikhina, Eugenia. Sin temor a Dios, ni a la justicia real: control social en Charcas a fines del siglo XVIII. La Paz: Instituto de Estudios Bolivarianos / Facultad de Humanidades y Ciencias de la Educación, 2001.

Cohen, Stanley. Visions of Social Control. Crime, Punishment and Classification. Cambridge: Polity, 1985.

Deflem, Mathieu. "Deviance and Social Control". The Handbook of Deviance. Ed. Erich Goode. Malden:Wiley-Blackwell, 2015.

"Bureaucratization and Social Control: Historical Foundations of International Police Cooperation”. Law \& Society Review 34.3 (2000): 739-778.

Di Liscia, Maria Silvia y Ernesto Bohoslavsky. Instituciones y formas de control social en América Latina. Una revisión. Buenos Aires: Prometeo, 2005.

Flaherty, David H. "Crime and Social Control in Provincial Massachusetts". The Historical Journal 24.2 (1981): 339-360.

Foucault, Michel. Vigilar y castigar. Nacimiento de la prisión. Buenos Aires: Siglo XXI, 2002. . Historia de la sexualidad.Volumen 1. México: Siglo XXI, 1991.

Foyster, Elizabeth. "Male Honour, Social Control and Wife Beating in Late Stuart England". Transactions of the Royal Historical Society 6 (1996): 215-224.

Gottfredson, Michael R. y Travis Hirschi. A General Theory of Crime. Stanford: Stanford University Press, 1990.

Greenberg, Douglas. "Crime, Law Enforcement, and Social Control in Colonial America”. The American Journal of Legal History 26.4 (1982): 293-325.

Griffiths, John. "The Division of Labor in Social Control". Toward a General 
Theory of Social Control. Selected Problems. Volumen 2. Ed. Donald Black. Orlando: Academic Press, 1984.

Griffin, Clifford S. "Religious Benevolence as Social Control, 1815-1860: The Mississippi Valley”. Historical Review 44.3 (1957): 423-444.

Hirschi, Travis. "In Defense of Self-Control”. Theoretical Criminology 4.1 (2000): 55-69.

Hirschi, Travis and David Rudisill. "The Great American Search: Causes of Crime, 1876-1976". Annals of the American Academy of Political and Social Science 423 (1976): 14-22.

Issel, William. "Americanization, Acculturation and Social Control: School Reform Ideology in Industrial Pennsylvania, 1880-1910”. Journal of Social History 12.4 (1979): 569-590.

Janowitz, Morris. "Sociological Theory and Social Control”. American Journal of Sociology 81.1 (1975): 82-108.

Kuhn, Lara Helena. Social Control and Human Nature: What is it We are Controlling? El Paso: LFB Scholarly Publishing LLC, 2009.

Marín Hernández, Juan José. “El control social y la disciplina histórica. Un balance teórico metodológico”. Revista de Historia de América 129 (2001): 31-71.

Mead, George Herbert. Espíritu, persona y sociedad. Desde el punto de vista del conductismo social. Buenos Aires: Paidós, 1972. . "Society". The Social Psychology of George Herbert Mead. Ed. Anslem Straus. Chicago: The University of Chicago Press, 1956. . "The Problem of Society - How we become Selves". The Social Psychology of George Herbert Mead. Ed. Anslem Straus. Chicago:The University of Chicago Press, 1956.

. "The Industrial Revolution". Movements of Thought in the Nineteenth Century. Ed. Merritt H. Moore. Chicago: University of Chicago Press, 1936.

."The Genesis of the Self and Social Control". International Journal of Ethics 35.3 (1925): 251-277.

Melossi, Dario. El Estado del control social. Un estudio sociológico de los conceptos de Estado y control social en la conformación de la democracia. México: Siglo XXI, 1992.

Messner, Steven F. y otros. "The Legacy of Lynching and Southern Homicide". American Sociological Review 70.4 (2005): 633-655.

Oliver Olmo, Pedro. "El concepto de control social en la historia social: estructuración del orden y respuestas al desorden”. Historia Social 51 (2005): 72-91.

Parsons, Talcott. The Social System. London: Routledge, 1991. . Essays in Sociological Theory. New York: The Free Press, 1954.

Phillips, David Charles. "Exploring Relations among Forms of Social Control: The Lynching and Execution of Blacks in North Carolina, 1889-1918”. Law \& Society Review 21.3 (1987): 361-374. 
Pound, Roscoe. Social Control Through Law. New Brunswick:Transaction Publishers, 1997.

Pratt, Travis C. y otros. "Key Idea: Hirschi’s Social Bond/Social Control Theory". Key Ideas in Criminology and Criminal Justice. New York: SAGE, 2010.

Reiss, Albert J. "Delinquency as the Failure of Personal and Social Controls". American Sociological Review 16.2 (1951): 196-207.

Ross, Edward Alsworth. Social Control. A Survey on the Foundations of Social Order. New York: The Macmillan Company, 1901.

Salvatore, Ricardo D. y Carlos Aguirre. Eds. The Birth of the Penitentiary in Latin America: Essays on Criminology, Prison Reform, and Social Control, 18301940. Austin: University of Texas Press, 1996.

Spierenburg, Peter. "Social Control and History". Social Control in Europe, 15001800. Volumen 1. Eds. Herman Roodenburg y Pieter Spierenburg. Ohio: The Ohio University Press, 2004.

Strauss, Anselm. "Introduction". The Social Psychology of George Herbert Mead. Ed. Anslem Straus. Chicago: The University of Chicago Press, 1956.

Szuchman, Mark D. "Disorder and Social Control in Buenos Aires, 1810-1860". The Journal of Interdisciplinary History 15.1 (1984): 83-110.

Thrupp, Sylvia L. "Social Control in the Medieval Town". The Tasks of Economic History 1 (1941): 39-52.

Weber, Max. Conceptos sociológicos fundamentales. Madrid: Alianza, 2014.

White, Harrison C. Identity and Control. Princeton: Princeton University Press, 2008.

Wiatrowski, Michael David. "Social Control Theory and Delinquency”. Tesis de doctorado en Estudios Urbanos, Portland State University, 1978. 